\title{
Dealing with wait times in prostate cancer
}

\author{
Bryan Donnelly, MD, FRCSC
}

See related article on page 243.

$\mathrm{T}$ his paper provides information which can be used to tackle the unacceptably prolonged time it takes to provide care for patients from the first suspicion of prostate cancer to treatment. ${ }^{1}$ The breakdown of intervals enables a more targeted approach to waiting times, which should offer better opportunities to significantly shorten delays.

As pointed out in the discussion, the introduction of a rapid access clinic in our own institution has greatly shortened the time from suspicion to diagnosis, as a first step in addressing wait times. This required a "paradigm shift" by all urologists in the city to move from an individual specialist intake to a central intake system. The importance of the relationship between the referring family doctor and specialist is maintained by ensuring that the patient is followed, and frequently treated by the specialist chosen by the family practitioner. This is felt to be a very important consideration. This system can be readily duplicated in most centres.

There is inevitable delay in accessing biopsy and pathological results. Access to ultrasound-guided biopsy can be hastened by using a dedicated centralized service. This also can promote greater expertise.

After diagnosis, with the veritable "smorgasbord" of treatment options available to patients today, delay at this point is inevitable. Patients need time to digest an overwhelming amount of information, and frequently require many consultations with different specialists. We are attempting to address this area by running multidisciplinary "prostate 101" classes for recently diagnosed patients and their partners/ family, and will analyze the results soon. These sessions are staffed by urologists, radiation oncologists, and medical oncologists, on a rotational basis, with an agreed set of slides and information.

The long interval from diagnosis to initiation of radiation is difficult to justify and, if it was in keeping with Canadian Association of Radiation Oncologists (CARO) suggested timelines, would provide significant reductions in delays. Access to radiation facilities is difficult to improve without significant financial investment. Access to radiation oncologists could, and should be improved. More radiation oncologists dedi- cated to uro-oncology, fee per item as opposed to salary, more emphasis on service in most centres, as opposed to research, all would help in this regard.

As pointed out in the paper, the medical implications of prolonged are not clear. Delays have been shown to cause patient and family psychological distress in this population. The view that "delays in medical treatment could have physical and stressful consequences" formed the basis of the Supreme Court of Canada decision in the Chaoulli versus Quebec case; this is an indication, from the highest court in the land, that prolonged wait times have negative implications.

While the numbers in this paper are small and preclude drawing significant conclusions, they do shed light on areas that should be examined more closely. With a willingness to adopt novel approaches, significant improvements would not be difficult to realize. We have an obligation to do all in our power to improve this situation for our patients.

Clinical Associate Professor, University of Calgary, Calgary, AB

Competing interests: None declared.

This paper has been peer-reviewed.

\section{Reference}

1. Stevens C, Bondy SJ, Loblaw DA. Wait times in prostate cancer diagnosis and radiation treatment. Can Urol Assoc J 2010;4:243-8.

Correspondence: Dr. Bryan Donnelly, 1011 Glenmore Trail SW, Calgary, AB T2V 4R6; donnelly@ ucalgary.ca 